Instructions for authors, subscriptions and further details:

http://redimat.hipatiapress.com

\title{
¿Cómo los Gestos de los Maestros Afectan a la Atención Visual de las Estudiantes durante el Discurso Matemático?
}

Danyal Farsani ${ }^{1}$ Adriana Breda ${ }^{2}$, and Gemma Sala Sebastià ${ }^{3}$

1) CIAE, Universidad de Chile, Chile

2) Universitat de Barcelona, Spain

3) Universitat Autònoma de Barcelona, Spain

Date of publication: October $24^{\text {th }}, 2020$

Edition period: October 2020-February 2021

To cite this article: Farsani, D., Breda, A., and Sala, G. (2020). ¿Cómo los gestos de los maestros afectan a la atención visual de las estudiantes durante el discurso matemático? REDIMAT - Journal of Research in Mathematics Education, 9(3), 220-242. doi: 10.17583/redimat.2020.5185

To link this article: http://dx.doi.org/10.17583/redimat.2020.5185

\section{PLEASE SCROLL DOWN FOR ARTICLE}

The terms and conditions of use are related to the Open Journal System and

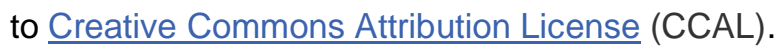


REDIMAT, Vol. 9 No. 3 October 2020 pp. 220-242

\section{How do Teacher's Gestures Affect Students' (Girls) Visual Attention During the Mathematical Discourse?}

Danyal Farsani

Universidad de Chile
Adriana Breda

Universitat de

Barcelona
Gemma Sala Sebastià

Universitat de

Barcelona

(Received: 16 February 2020; Accepted: 22 June 2020; Published: 24

October 2020)

\section{Abstract}

Engaging with students and building rapport is critical in order for teachers to teach classes, communicate core concepts and emotionally connect with their students in regard to the cultural dynamism that occurs in the classrooms through its dialogic approach. This paper reports a study that was conducted in an upper primary school in Santiago, Chile, where a sample of 33 randomly selected female students wore a mini video camera mounted on eyeglass in their mathematics lessons. Using Google Images, we automatically and objectively looked form frames from the recordings where the classroom teacher appeared in the students' visual field. The results show that students significantly paid more visual attention in their mathematics lessons when the teachers' discourse was accompanied by gestures. In addition, there were differences between high and low achieving girls and how they were visually engaged during teachers' instructional information at different times of the lesson. Furthermore, there were differences between how introverts and extroverts were visually engaged while the teacher was gesturing. The findings of this study will have broad significance for building rapport and improving professional teaching practices.

Keywords: Visual attention, classroom interaction, gestures, eye gaze, didactics 


\section{¿Cómo los Gestos de los Maestros Afectan a la Atención Visual de las Estudiantes Durante el Discurso Matemático?}

$\begin{array}{lll}\text { Danyal Farsani } & \text { Adriana Breda } & \text { Gemma Sala Sebastià } \\ \text { Universidad de Chile } & \begin{array}{l}\text { Universitat de } \\ \text { Barcelona }\end{array} & \begin{array}{l}\text { Universitat de } \\ \text { Barcelona }\end{array}\end{array}$

(Recibido: 16 Febrero 2020; Aceptado: 22 Junio 2020; Publicado: 24 Octubre 2020)

\section{Resumen}

Interaccionar con los estudiantes y construir una buena relación es fundamental para que los maestros enseñen, comuniquen conceptos básicos y se conecten emocionalmente con sus estudiantes. Este artículo reporta un estudio que se llevó a cabo en una escuela de educación primaria en Santiago de Chile, donde una muestra de 33 estudiantes (niñas), seleccionadas al azar, llevaban una minicámara de vídeo anexada en sus gafas en el momento que participaban en las clases de matemáticas. Usando imágenes de Google, se buscaron, de forma automática y objetiva, registros de las grabaciones en las que el profesor de la clase aparecía en el campo visual de las estudiantes. Los resultados muestran que las estudiantes prestaron mucha más atención visual en las clases de matemáticas cuando el discurso de los profesores iba acompañado de gestos. Además, hubo diferencias entre las niñas con alto y bajo rendimiento y en cómo se involucraron visualmente durante la instrucción de los maestros en diferentes momentos de la lección. También, se presentaron diferencias en relación con cómo las alumnas introvertidas y las extrovertidas se involucraban visualmente mientras el maestro hacía gestos. Los resultados de este estudio son muy importantes para poder construir una buena relación interaccional entre profesor-alumno y mejorar las prácticas profesionales de enseñanza.

Palabras clave: Atención visual, interacción en el aula, gestos, mirada, didáctica 



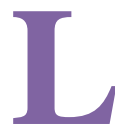

as interacciones son un fenómeno social importante en las aulas. Las formas en que un maestro interactúa con sus alumnos son fundamentales para construir una buena relación y enseñar los más variados tipos de contenidos. Actualmente, la interacción en el aula y la construcción de una relación son elementos importantes de los patrones de las relaciones sociales, algo que tradicionalmente, hace unos años, no se consideraba importante para los procesos educativos. La investigación sobre la interacción en el aula ha tenido una larga tradición en la investigación educativa (Pogue \& Ahyun, 2006; Andersen, 1978). Algunos de los enfoques teóricos relacionados con la Educación Matemática, como en el Enfoque Ontosemiotico (EOS) (Godino, Batanero, \& Font, 2007; 2019; Breda, Font, \& Pino-Fan, 2018) o bien la Teoría Antropológica de lo didáctico (TAD) (Chevallard, 1999; Bosch \& Gascón, 2009) desarrollaron herramientas para analizar los procesos interactivos que ocurren en un proceso de instrucción matemático, ayudando al profesor, en particular, a reflexionar y gestionar dichos procesos.

Desde el punto de vista metodológico, la investigación sobre los procesos interactivos en el aula involucra, con frecuencia, el uso de una cámara grabadora puesta en un trípode en la parte posterior del aula con el objetivo de capturar la relación que hay entre los estudiantes y el maestro. De esta manera, las investigaciones incorporan la perspectiva de una tercera persona para dar cuenta de los procesos de enseñanza y aprendizaje, es decir, las interacciones entre estudiantes y maestros son, generalmente, analizadas desde el punto de vista de una tercera persona. Con este método de análisis, la investigación resulta ser más bien de tipo descriptivo y subjetivo (Catalán, 2018; Farsani \& Mendes, in press).

Como se ha mencionado anteriormente, los datos que se capturan con este tipo de cámara son la perspectiva de una tercera persona, que tiene la intención de describir la interacción entre el profesor y el alumno. Una consecuencia de utilizar este tipo de instrumento es que los datos no surgen ni de las perspectivas del profesor, ni de los alumnos, sino de una cámara de vídeo, que es un objeto no animado cuya presencia no es natural en el aula. Por lo tanto, entendemos que existe la necesidad de que los maestros identifiquen acciones pedagógicas en forma de prácticas de enseñanza y aprendizaje mediante la aplicación de otra perspectiva tecnológica, como, por ejemplo, la tecnología visual (Prieto, Sharma, \& Dillenbourg, 2015). 


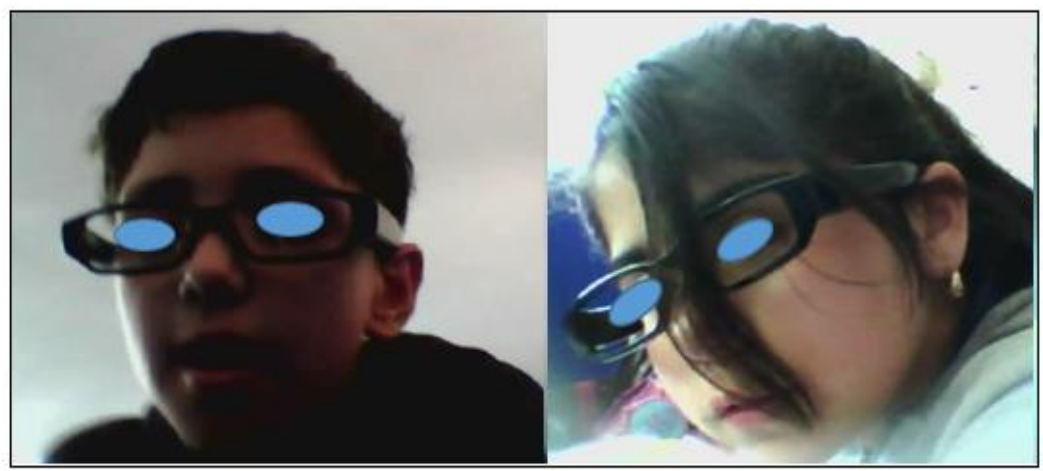

Figura 1. Mini cámara montada en las gafas de los estudiantes.

En este estudio, proponemos incorporar la perspectiva de una primera persona en los vídeos. Para tener dicha perspectiva, estas grabaciones de vídeo interactivas se consiguen por medio del uso de mini cámaras de vídeo que han sido montadas en las gafas de los estudiantes. Se trata de dispositivos tecnológicos ampliamente utilizados recientemente en investigaciones educativas, ya que son baratos y disponibles fácilmente.

La tecnología visual puede proporcionar nuevas oportunidades para detectar patrones de interacción sutiles pero importantes en las aulas. Estas gafas permiten a los investigadores registrar y volver a ver lo que los estudiantes observan y perciben desde su propio punto de vista particular, que es la opinión de una primera persona. Esto incluye cualquier aspecto, desde lo que está escrito en la pizarra o en sus cuadernos, la percepción de gestos y comunicación no verbal realizadas por el maestro hasta cuánta atención visual prestan al maestro o a otros estudiantes. La Figura 1 muestra una mini cámara montada en las gafas de los estudiantes.

En este artículo, argumentamos que las grabaciones de vídeo en primera persona son un complemento de la observación regular en el aula que puede mejorar el conocimiento de los maestros sobre su impacto en la atención de los estudiantes y así buscar estrategias para mejorar la interacción entre profesor y alumno (Farsani \& Mendes, in press).

El objetivo de este artículo es evidenciar la relación entre la involucración visual del estudiante con su profesor de matemáticas y ciertos aspectos que podrían tener influencia en su aprendizaje. Utilizando los datos obtenidos a partir de las vídeo cámaras montadas en las gafas usadas por 33 estudiantes, 
en este artículo exploramos, no sólo su atención visual en las clases de matemáticas, sino también, de qué modo las alumnas, en función de su rendimiento, más elevado y menos elevado, y de si son introvertidas o extrovertidas, están visualmente involucradas con el profesor de matemáticas. Asimismo, se analiza la diferencia de atención visual de las estudiantes en diferentes momentos de la lección (durante un período de 90 minutos).

A continuación, se presenta una breve revisión de la literatura acerca del uso de los gestos en la investigación educativa, en particular, los gestos utilizados en el discurso matemático y aclaraciones sobre la mirada visual e interacción no verbal entre niños y niñas. Además, se explica la metodología de recolección y análisis de los datos, los resultados y las consideraciones de este estudio.

\section{Gestos en la Investigación Educativa}

El uso de los gestos en la investigación educativa puede presentar distintas definiciones. Una de ellas es la definición de un tipo de lenguaje llamado lenguaje no verbal. De forma resumida, el lenguaje no verbal se "refiere al funcionamiento comunicacional de la actividad corporal, gesto, expresión y orientación facial, postura y espacio, tacto y olfato, y de aquellos aspectos de la expresión que pueden considerarse aparte del contenido referencial de lo que se dice" (Kendon, 1983, p.3). En la literatura, por ejemplo, el término 'cinética' se utiliza para encontrar cualquier movimiento corporal, como gestos, expresiones faciales, etc., como medio de comunicación. Hockings (1995, p. 509), en particular, define la cinética como "una ciencia de la comunicación por gestos humanos". En un terreno similar, Collier (1995, p. 235) define la cinética por medio del "significado de la expresión corporal". Otro término comunicativo no verbal ampliamente utilizado es el gesto. El término 'gesto' se usa en un sentido amplio, como un movimiento físico de una parte del cuerpo (por ejemplo, manos, brazos, ojos y cara) (Kendon, 1983; Maschietto \& Bussi, 2005) en situaciones comunicativas (Kendon, 1997; Streeck, 1988).

Esta sección discute diferentes formas de gestos que se emplean generalmente en la comunicación humana. Hay gestos que se producen de forma independiente a sus unidades de habla correspondientes (emblemas) versus gestos que se producen en coordinación con el habla (gestos que 
acompañan al habla). La primera categoría de gestos mencionada, a menudo, se denomina "gestos independientes del habla" (Knapp \& Hall, 2006) también se conocen como "emblemas" (Ekman, 1976) o "gestos autónomos" (Kendon, 1983; 1985). Los emblemas son "actos no verbales que tienen una traducción verbal directa, generalmente consiste en una palabra o dos o una frase" (Knapp \& Hall, 2006, p. 226). Estos son gestos convencionales que tienen un significado común en un contexto cultural particular.

En muchas culturas, por ejemplo, el gesto de "puño cerrado con el pulgar hacia arriba" (ver Figura 2) es el signo de "aprobación", que fue cooptado por Facebook como el signo de "me gusta", presumiendo que no se necesitan otros modos de lenguaje para que su significado se entienda. Sin embargo, en Francia y en la mayoría de los países occidentales, el signo de "pulgar hacia arriba" también tiene el significado de "campeón" o "súper", en base al signo romano para salvar la vida de un gladiador (Corbell, 1997). Además, este mismo gesto puede significar también "iVete al infierno!" o una "invitación sexual explícita" en la cultura iraní (Galanti, 2004). Matemáticamente, tiene el significado de "uno" para los italianos, "cinco" para los japoneses (Koechlin, 1992) y para los británicos-iraníes de herencia persa, significa "sesenta" (Farsani, 2015b). Así pues, los emblemas son gestos que se envían y reciben conscientemente con un significado específico que puede variar de una cultura a otra (Ekman \& Friesen, 1969). Si bien los emblemas se pueden entregar en absoluto silencio, los gestos espontáneos se producen en sincronía con el flujo del habla. La categoría de gestos básicos de McNeill (1992) se basa en el habla y gestos que se manifiestan mediante movimientos corporales espacio-temporales. Su categoría de gestos tiene en cuenta los gestos que se producen de forma dependiente con sus unidades de habla correspondientes. Este autor categorizó cuatro formas diferentes de gestos con las manos donde cada forma tiene una función diferente en la comunicación humana. Hay gestos 'deícticos', 'vencidos', 'icónicos' y 'metafóricos'.

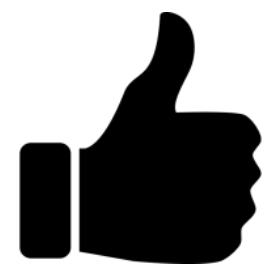

Figura 2. El signo de "ok" o "pulgares arriba". 
Los gestos deícticos consisten en movimientos indicativos que a menudo se producen con un dedo índice extendido, pero también, con los otros dedos de la mano o con la mano entera y sirven para indicar ubicaciones, direcciones, personas u objetos, estén o no presentes en el entorno. Los gestos deícticos no transmiten información perceptiva o de acción, solo se usan para señalar objetos y ubicaciones. Al igual que los gestos deícticos, los gestos de ritmo no tienen ningún significado.

Los gestos de ritmo se coordinan rítmicamente con el discurso y los movimientos frecuentemente son cortos y rápidos. Los gestos de ritmo no transmiten el significado semántico del habla, sino que están "alineados con los patrones de prominencia prosódica en el habla" (Gullberg, 1998, p. 51). Este tipo de gesto, a menudo, es más notable cuando es producido por músicos o políticos. El tercer tipo de gesto es el icónico. Los gestos icónicos se asemejan a objetos concretos o acciones que "representan el contenido semántico directamente a través de la forma o trayectoria de movimiento de las manos (p. ej. trazando un triángulo en el aire para significar triángulo)" (Alibali \& Nathan, 2012, p. 251). Los gestos icónicos transmiten el significado semántico a través de las imágenes creadas por el hablante. Finalmente, la última clasificación de gestos son los metafóricos. Los gestos metafóricos son similares a los gestos icónicos en el sentido de que transmiten el contenido semántico a través de la metáfora. Las metáforas gestuales "son como gestos icónicos en el sentido de que son pictóricos, pero el contenido pictórico presenta una idea abstracta en lugar de un objeto o evento concreto. El gesto presenta una imagen de lo invisible: una imagen de una abstracción" (McNeill, 1992, p. 14). Los gestos icónicos y metafóricos son imaginarios, presentan una imagen que es nula / presente en el entorno.

\section{Gestos en el Discurso Matemático}

Una de las razones por las que estamos tan interesados en la comunicación no verbal es por su importancia en la enseñanza y el aprendizaje, ya que a menudo la investigación no aborda el papel de la comunicación no verbal en los entornos educativos (Lemke, 1998). Además, Roth (2002, p. 365) ha observado que, "[...] aquí existe muy poca investigación educativa relacionada con el papel de los gestos en el aprendizaje y la enseñanza, particularmente en las áreas temáticas que se han caracterizado por tratar temas abstractos como las ciencias experimentales y las matemáticas". 
En contrapartida, en los últimos veinte años, el papel de los gestos en la Educación Matemática ha recibido considerable atención (Sfard, 2009). Se ha demostrado que los gestos reflejan el pensamiento de los hablantes sobre conceptos matemáticos y se ha sugerido que pueden desempeñar un papel crucial en la transmisión del conocimiento en las interacciones de enseñanza / aprendizaje (Farsani, 2015b). Sin embargo, un gesto, como cualquier otro modo de comunicación, es un artefacto cultural. La frecuencia de los gestos y los significados que suelen transmitir varían según las culturas (para una discusión de los gestos árabes y persas, ver Barakat, 1973; Sparhawk, 1976; 1981) y dependen de factores sociales y culturales (Núñez \& Sweetser, 2006). En la Educación Matemática, la investigación sobre gestos rara vez ha tenido en cuenta el contexto cultural. En este artículo, consideramos cómo los gestos contribuyen a la producción de un aumento de la atención visual y a la participación visual de las estudiantes en sus lecciones de matemáticas en el contexto de la capital chilena.

En el aula de matemáticas, los gestos son recursos visuales que pueden ayudar a los estudiantes a resolver dudas sobre términos matemáticos específicos, además de ayudarlos a construir o bien a generar un determinado significado (Alibali, 1999). Por ejemplo, Castellón (2007) utilizó una serie de observaciones etnográficas en las aulas de matemáticas para ilustrar cómo los gestos de los estudiantes hispanos en Los Ángeles funcionaban como ayudas para superar obstáculos en la comprensión de términos matemáticos, como, por ejemplo, la noción de recta perpendicular. En este caso, a menudo se usaba un gesto para acentuar, y algunas veces reemplazar, las expresiones verbales. El autor muestra que un "gesto sirvió como una herramienta visual que permitió a los estudiantes comunicar sus procesos de pensamiento sin limitarse a terminología como vertical, horizontal, paralela o perpendicular" (Castellón, 2007, p.163).

Se han encontrado gestos para ayudar a los maestros y profesores a expresar sus explicaciones en el aula (Pier \& Nathan, 2016). Alibali y Nathan (2007) observaron que el uso de gestos espontáneos de manos y brazos, junto con el discurso expresado por el profesor de matemáticas, hizo que los estudiantes no comprendieran los significados matemáticos pretendidos. Sin embargo, Farsani (2016), argumenta que los gestos que hacen los maestros pueden facilitar la comprensión de los conceptos por parte dos alumnos, más aún cuando el idioma utilizado en la instrucción no es la lengua oficial de los 
estudiantes, situación que suele pasar con los actuales procesos inmigratorios.

Los gestos que acompañan las expresiones verbales pueden apoyar la enseñanza de los significados matemáticos en aulas multilingües o que presentan alta diversidad lingüística. Farsani (2015b) mostró que los gestos producidos en las lecciones de matemáticas por el mismo hablante cambiaban según el idioma utilizado. Por ejemplo, la forma y la función de los gestos vinculados a una discusión sobre el concepto matemático de "power" en inglés, traducido en español como "exponente" transmitía una metáfora conceptual diferente que cuando se presentó la idea en persa. Tanto el profesor como los alumnos apuntaron con los dedos hacia la posición de superíndice de la notación en inglés, pero en persa, la descripción del mismo concepto matemático fue acompañado con un gesto de puño cerrado, que sugiere poder físico. Mientras que el término inglés, "exponente", no produjo tal asociación, la palabra persa utilizó en el gesto un significado alternativo de poder para apoyar una conexión metafórica. Estos ejemplos sugieren que un lenguaje hablado puede indicar diferentes metáforas cuando va acompañado de gestos, agregando así capas de significado a la información que se transmite. En esta situación, los diferentes idiomas hablados por el mismo hablante activaron diferentes interpretaciones conceptuales del mismo concepto matemático.

\section{Mirada Visual e Interacción no Verbal entre Niños y Niñas}

La interacción no verbal, frecuentemente, se emplea como un instrumento para el manejo del comportamiento en contextos educativos (Rogers, 2015). Sin embargo, el papel de la atención visual en los contextos de aulas de clase es relativamente nuevo (Prieto, Sharma, \& Dillenbourg, 2015).

Raca y Dillenbourg (2013), por ejemplo, crearon un sistema para evaluar la atención en el aula. García y Hannula (2015) implementaron el seguimiento ocular para explorar cómo las interacciones no verbales de los docentes (como los gestos que hacen los docentes) afectan la atención visual de los estudiantes. Por supuesto, como lo sugirieron García y Hannula (2015), se deben realizar más estudios para ver qué tipos de gestos pueden beneficiar a los estudiantes a recordar las instrucciones de los maestros.

Por lo tanto, en este artículo exploramos las diferencias de atención visual entre niñas extrovertidas e introvertidas y las diferencias entre las que 
presentan un alto o bajo rendimiento en matemáticas. Creemos que explorar este aspecto puede plantear cuestiones importantes para pensar sobre las interacciones entre maestros y estudiantes, así como, el papel de estas interacciones en los procesos de aprendizaje.

Es interesante notar que hay factores verbales que pueden tener un efecto directo en la atención visual y el comportamiento no verbal de los estudiantes. En un estudio anterior, Farsani (2015a) observó cómo estudiantes bilingües británico-iraníes (con el inglés como segundo o tercer idioma) 'cambiaron' sus espacios interpersonales, es decir, los ángulos de giro que realizaban con su cuerpo y la frecuencia con que establecían contacto físico. Se identificó, además, que cuando se usaba el inglés como lengua de instrucción, los estudiantes tenían más probabilidades de comunicarse sobre temas matemáticos relacionados con el aula. No obstante, cuando se usó el persa, se crearon espacios interpersonales más cercanos entre los estudiantes (el lenguaje es parte integral de una cultura que ayuda a desarrollar rasgos culturales en el aula). Además, mientras que los niños mantuvieron la misma postura, curiosamente, las niñas hicieron un giro en la postura, generando más contacto visual entre sí mientras discutían tareas matemáticas.

Hasta donde sabemos, nunca se ha llevado a cabo un estudio para explorar las diferencias entre alumnos y alumnas con bajos y altos logros y cómo esto afecta su atención visual en las lecciones de matemáticas. Además, en esta investigación también se explora, si las características individuales de ser introvertido o extrovertido tienen algún efecto en la atención visual.

\section{Metodología: Proceso de Recolección de Datos}

Los datos utilizados en este artículo son parte de un conjunto de datos más amplio, que provienen de un estudio para investigar los patrones inconscientes de comportamiento en la interacción entre el maestro y los alumnos. El foco de nuestra atención es capturar y analizar los patrones de mirada entre los estudiantes y el maestro de la clase para abrir la "caja negra" de las prácticas interactivas que ocurren en el aula desde la perspectiva de la primera persona. En este artículo, presentamos una muestra de 33 estudiantes (33 niñas) seleccionadas al azar entre todas las candidatas con una edad promedio de 10,5 años. La razón de que la participación en el estudio fuera exclusivamente femenina se debió a consideraciones éticas, ya que para 
llevar a cabo la investigación fue necesaria la entrega de formularios de consentimiento ético por parte de los padres y tutores de los estudiantes y, casualmente, de todos los estudiantes invitados a participar del estudio, estas 33 alumnas fueron las que primero entregaron sus formularios de consentimiento mostrando su disponibilidad a participar en este estudio. A partir de conocer a las estudiantes participantes, por medio de la ayuda del psicólogo escolar, se pudo crear un perfil de cada alumna. Obtuvimos información adicional individual de cada estudiante, concretamente, sobre si son consideradas como introvertidas o extrovertidas, si presentan necesidades especiales, si son académicamente de alto rendimiento (presentan evaluación por encima del promedio) o de bajo rendimiento (presentan evaluación por debajo del promedio). Esta información nos permitió distinguir a cada alumna en función de sus diferencias individuales, en relación con sus características personales y académicas.

El proceso de recolección de datos fue realizado durante las clases de matemáticas de dos escuelas (una pública y otra mixta o concertada) ubicadas en una zona económica y socialmente vulnerable de Santiago de Chile. La pública tenía una subvención del $100 \%$ por parte del gobierno, y la mixta o concertada tenía una subvención del $90 \%$ por parte del gobierno. En ambas escuelas, la proporción de estudiantes femeninas era más alta que la de los estudiantes masculinos.

Cada lección consistió en dos periodos de aula consecutivos de 45 minutos (90 minutos), donde se pidió al maestro de aula y a una muestra de 33 estudiantes seleccionadas al azar (6 estudiantes cada día) que utilizasen una mini cámara de vídeo montada en gafas especiales. En total, hubo 45 horas de clases grabadas, las cuales fueron, mayoritariamente, de tipo magistral, es decir, la configuración del aula se daba con el maestro explicando el contenido y los alumnos atentos a su explicación.

Al montar las cámaras en las gafas de los alumnos, podemos obtener una mejor perspectiva de la clase, tal como la ve el alumno. En total, obtuvimos casi 45 horas de grabaciones interactivas de las estudiantes. Estas cámaras de vídeo tenían una calidad de grabación de treinta frames por segundo (30 fps) (un frame es la unidad mínima, en forma de imagen estática, en que se puede descomponer una secuencia de vídeo); para cada vídeo, se tomó una muestra de un frame cada segundo y se procesó para detectar la presencia de caras. Al final de cada día, las grabaciones se descargaban manualmente en un ordenador. Montar una mini cámara de vídeo no es un gran cambio, ni una 
gran perturbación para los estudiantes. Es solo un pequeño complemento para las gafas. En nuestra experiencia, después de unos minutos, las estudiantes olvidaron por completo que estaban usando las gafas, siendo un instrumento que pasó a formar parte de la cultura del aula. En otras palabras, estas gafas se "encarnaron" y formaron parte de los estudiantes. Son unas gafas muy ligeras y asequibles. Por lo tanto, podrían ser ampliamente utilizadas si se demostrara que son útiles para maestros y estudiantes.

\section{Procesamiento Visual Automatizado}

Al incorporar tecnología visual para observar la interacción en el aula, se pueden proporcionar nuevas oportunidades para detectar patrones sutiles pero importantes en las clases. Una novedosa peculiaridad de este artículo es su enfoque metodológico en el análisis de datos visuales, ya que utilizamos un enfoque automatizado y objetivo para analizar los frames.

Cada frame muestreado (cada frame que representa un segundo) se envió a través del software Google Images. Se usaron fotos para detectar la presencia de caras. Insertamos fotos del profesor del aula, y Google Images identificó de forma automática y objetiva, de cada uno de los frame capturados por las alumnas, todos aquellos donde aparecía una imagen del maestro. Se analizaron un total de 5064 frames.

Estábamos principalmente interesados en los casos en que las estudiantes mantenían su atención visual en el maestro. Hubo ocasiones en las que había más de dos caras presentes en el mismo frame, por ejemplo, el maestro y otra persona que acababa de llegar tarde a clase. En estos casos, decidimos descartar el frame ya que la atención visual de la alumna pudo haberse fijado en la alumna que llegaba tarde y no en el profesor.

Hubo otros casos en los que descartamos deliberadamente los frames y no los tuvimos en cuenta en el análisis. Esto incluyó casos en los que la claridad de los frames era baja o borrosa y, por lo tanto, no se podía discernir si el maestro estaba mirando al estudiante o no. El hecho de poner en práctica todas estas medidas estrictas hizo que nuestra interpretación del análisis de los frames fuera más efectiva.

Una vez que Google Image detectaba la cara de un maestro en un frame (capturado por las gafas de las estudiantes), este frame recibía un número de identificación único (ver Figura 3a) y era examinado manualmente para observar las variables no verbales - orientación corporal, gestos, caminando, 
mirando por debajo de los anteojos, señalando, señalando con los labios, posición de defensiva, leyendo, etc. Por ejemplo, nos preguntábamos: En este frame el maestro ¿estaba mirando a las estudiantes de forma directa? o ¿estaba gesticulando, señalando o apuntando hacia las estudiantes?

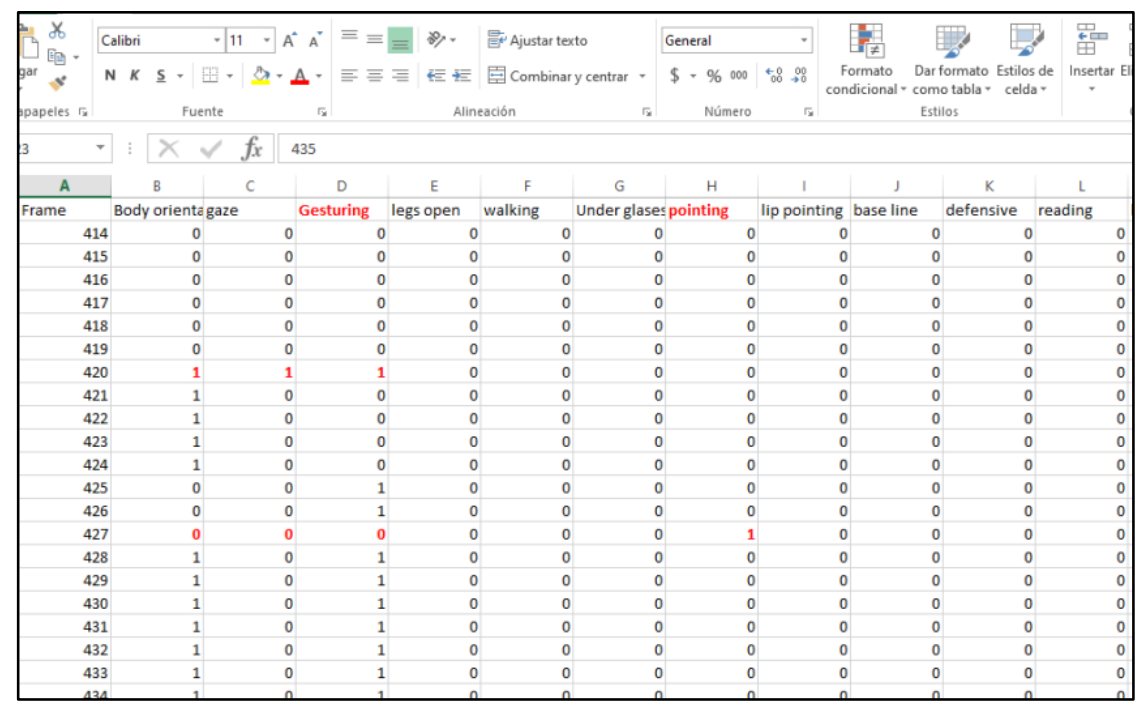

Figura $3 a$. Examen de variables no verbales.

Este proceso manual se realizó en Excel insertando ceros (0) y unos (1) — entendiéndolo como no sucedió, versus sí sucedió- y luego, se realizó un análisis basado en estadística descriptiva para medir su importancia. Además de las variables no verbales mencionadas en el párrafo anterior, también se consideraron otras variables no verbales, como, por ejemplo, si un maestro estaba usando el escritorio como una barrera entre él y las estudiantes, escribiendo en la pizarra, o si el maestro estaba caminando por la clase o mantenía una posición estática. Por ejemplo, examinemos los frames de números 420 y 427, respectivamente (cada uno representa un segundo) capturados por las gafas de una alumna. La Figura 3 b representa el frame 420 y la Figura 3c representa el frame 427, que se pueden observar registrados cuantitativamente en la figura $3 \mathrm{a}$. En el frame 420, se percibe que el maestro a) orienta su cuerpo hacia esta estudiante en particular, b) mira a la estudiante, y c) gesticula. En el frame 427, el maestro no parece estar mirando 
directamente a la alumna, ni orientando su cuerpo hacia ella, sino que parece estar señalando.

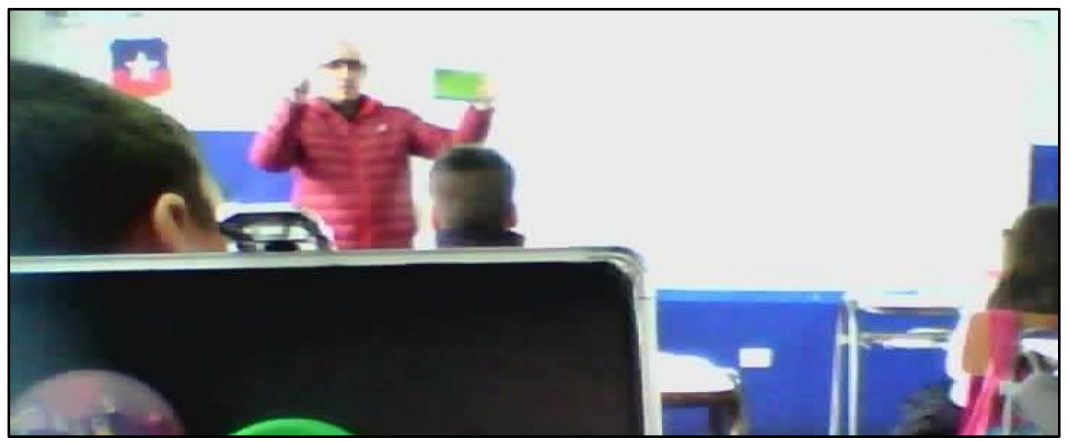

Figura 3b. Observando al maestro mientras gesticula

Estas descripciones cuantitativas se han realizado basándonos en el conteo de la frecuencia de las variables no verbales (conforme la Figura 3a) representadas en cada frame.

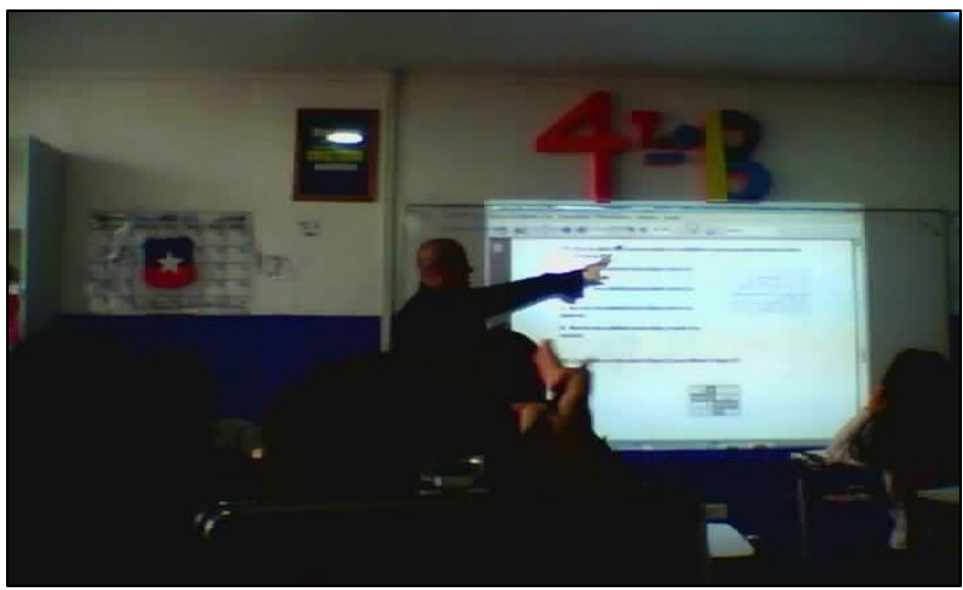

Figura 3c. Observando al maestro mientras señala 


\section{Análisis de los Datos}

Gracias a la metodología utilizada en este estudio se puede identificar, por medio de algunas de sus características (por ejemplo, introvertidos o extrovertidos, o aquellos con diferentes habilidades de aprendizaje), qué estudiante está mirando al profesor durante el transcurrir de la clase. Por esta razón, en esta sección ofreceremos cuatro tipos diferentes de análisis, y cada análisis significará un enfoque diferente para mostrar la interacción en el aula. Primero, se expone la cantidad de estudiantes visualmente comprometidas con su profesor durante la lección de 90 minutos. En segundo lugar, se revela la importancia de los gestos y los gestos de los maestros (cuando el profesor hace gestos y señala) para mostrar cuántas veces las estudiantes aprecian las señales no verbales de sus maestros. En tercer lugar, examinamos las formas en que las estudiantes, distinguiendo si son de mayor o menor rendimiento escolar, se involucran visualmente y miran al profesor durante el tiempo de la lección.

\section{Atención Visual de las Estudiantes Durante su Clase de Matemáticas}

La Figura 4 ilustra una visión general de cuánto fue la participaron visual de estas 33 estudiantes en su clase de matemáticas. Los datos que se aportan en esta sección provienen de casi 50 horas de grabaciones de vídeo interactivas por medio de las cámaras montadas en las lentes de las estudiantes. En los 10 primeros minutos de la clase se han capturado 1355 frames de la interacción, en particular, es el momento en que las estudiantes tienen una mayor atención visual en su maestro. Es interesante observar que la atención visual disminuye drásticamente en los minutos siguientes (de 10-20 y 20-30), pero vemos un ligero aumento en la atención visual de las estudiantes al cabo de 30-40 minutos. Por supuesto, debido a la naturaleza de los datos que tenemos, no se puede explicar la razón de la baja o el aumento repentinos de esta atención visual. Lo interesante es notar que, después del minuto 40, las estudiantes comienzan a desconectarse bruscamente y pierden su interés (o al menos su atención visual en su maestro). ES decir, después del minuto 40, las estudiantes empiezan a perder interés visual en su maestro, esta falta de interés es gradual llegando a bajar hasta los 50 frames en el minuto 90. En nuestra observación, las estudiantes no volvieron a recuperar su pico de 
compromiso visual que, como se ha mencionado, había sido de 1355 frames en los primeros 10 minutos de la clase.

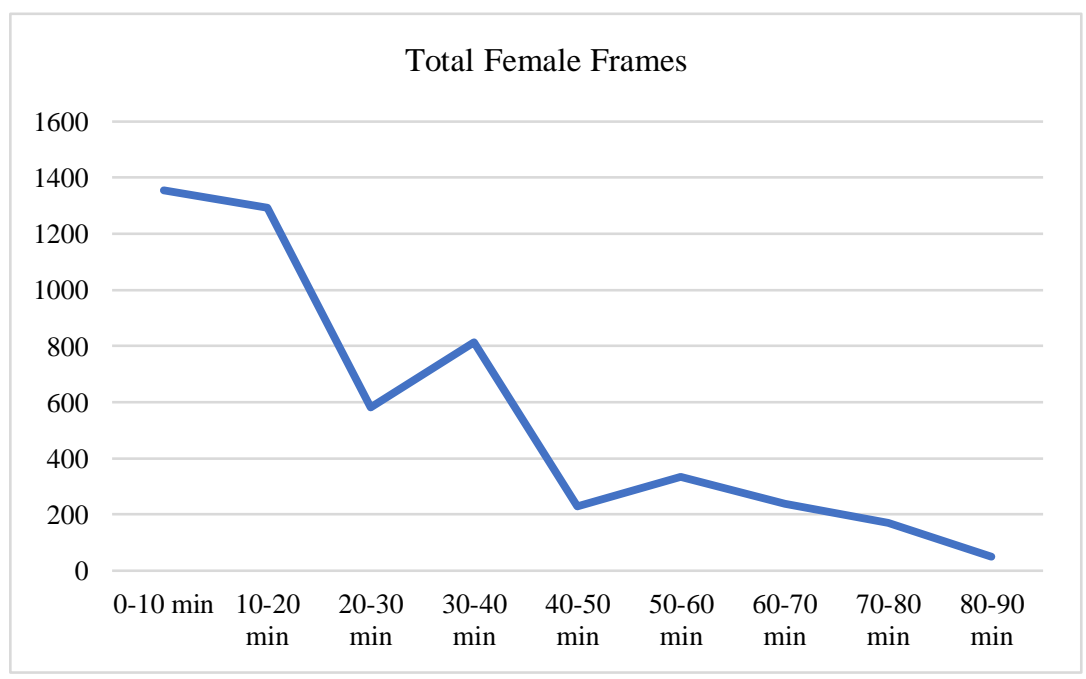

Figura 4. Examinando la atención visual de las estudiantes durante un intervalo de 90 minutos

Como mencionamos en la revisión de la literatura de este artículo, existen gestos para ayudar a los estudiantes a construir significados matemáticos (Farsani, 2015b) o para transmitir un significado matemático (Farsani, 2015b). Es interesante observar que, hasta la fecha, no se ha realizado ningún estudio que muestre cuántos gestos $\mathrm{y}$, en particular, qué señales pueden facilitar que los maestros conecten con los estudiantes y mantengan su atención visual.

Vale la pena analizar la perspectiva que ofrece la Figura 5 para poder establecer cierta causalidad. Como observamos en el gráfico anterior (Figura 4), hubo 1355 frames que muestran que las estudiantes prestaron atención visual a su maestro en los primeros 10 minutos de la lección. En esos mismos primeros 10 minutos, también hubo 775 frames que muestran que el maestro estuvo gesticulando (gestos metafóricos, icónicos y de ritmo) y 587 frames donde el maestro parece que estuvo haciendo acciones de señalar. Cabe subrayar que cada frame puede tener múltiples variables realizadas a la vez por el maestro en ese mismo momento. Por ejemplo, un maestro podría estar 
gesticulando con una mano mientras señala los escritos en la pizarra con otra mano. Por lo tanto, en los primeros 10 minutos de la lección, los gestos y acciones de señalar del maestro se usaron como instrumentos para captar la atención visual de las estudiantes.

Durante los siguientes 10 minutos los gestos realizados por el maestro parece que tuvieron muy pocos efectos en captar la atención visual de las estudiantes y, en cambio, señalar con el dedo sí que consiguió captarla. En el mundo de la política, el apuntar a menudo transmite una connotación negativa, sin embargo, parece que en el caso de los maestros puede ser utilizado como un gran instrumento para atraer la atención visual de sus estudiantes durante la clase.

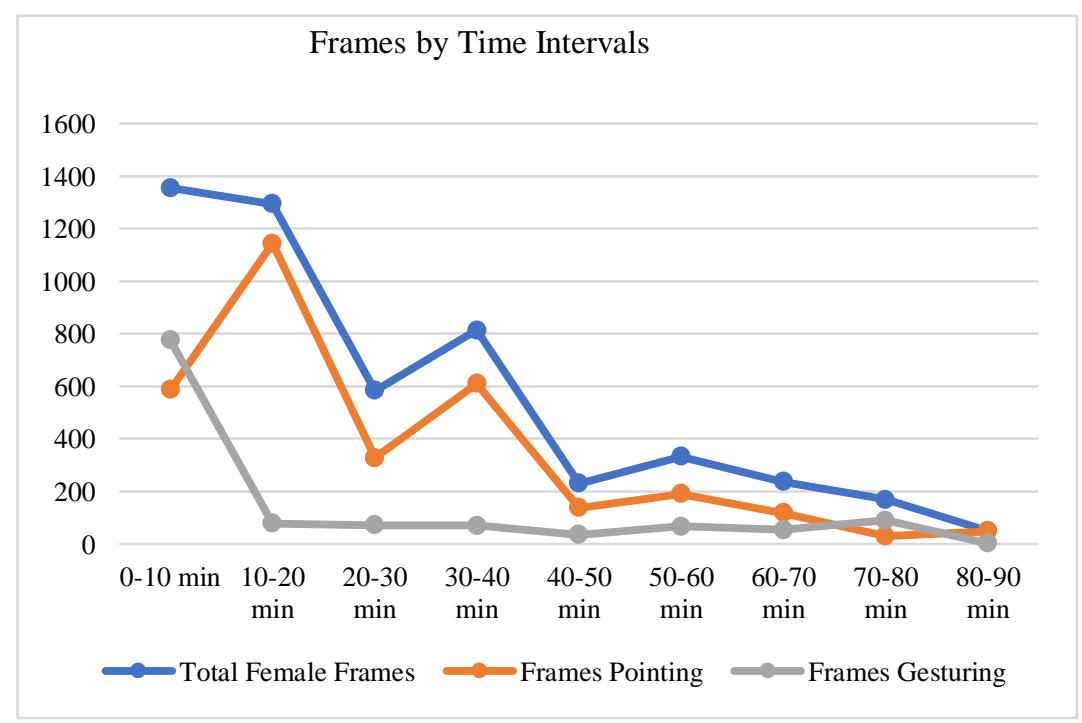

Figura 5. Los efectos de los gestos y las acciones de señalar del docente sobre la atención visual de las estudiantes

\section{Estudiantes de Mayor y Menor Rendimiento y su Atención Visual}

En nuestro estudio, conforme lo explicado en el apartado de la metodología, se creó un perfil de cada una de las estudiantes. En esta sección, compararemos las estudiantes en relación con sus logros académicos, es 
decir, a partir de un promedio de calificaciones Grade Point Average (GPA) alto / bajo, se analizará cómo se involucran visualmente en el aula. Para clasificar el GPA alto/bajo, se utilizó la referencia de una "puntuación promedio" adjudicada a cada lección. Las alumnas que obtuvieron una calificación más baja del promedio se colocaron automáticamente en GPA bajo y aquellas estudiantes que obtuvieron calificaciones más altas que el promedio, se categorizaron con el GPA alto.

La Figura 6 ofrece otra perspectiva sobre la naturaleza de la interacción en el aula. Podemos ver claramente que tanto las estudiantes con un GPA alto como las estudiantes con un GPA bajo mantienen el mismo patrón de atención visual. La única diferencia es que el compromiso visual con el maestro de las estudiantes con un GPA alto tiende a realizarse más durante la primera mitad de la lección. En la segunda mitad, tanto las que obtienen mejores resultados como las que no, tienden a mirar al maestro por igual.

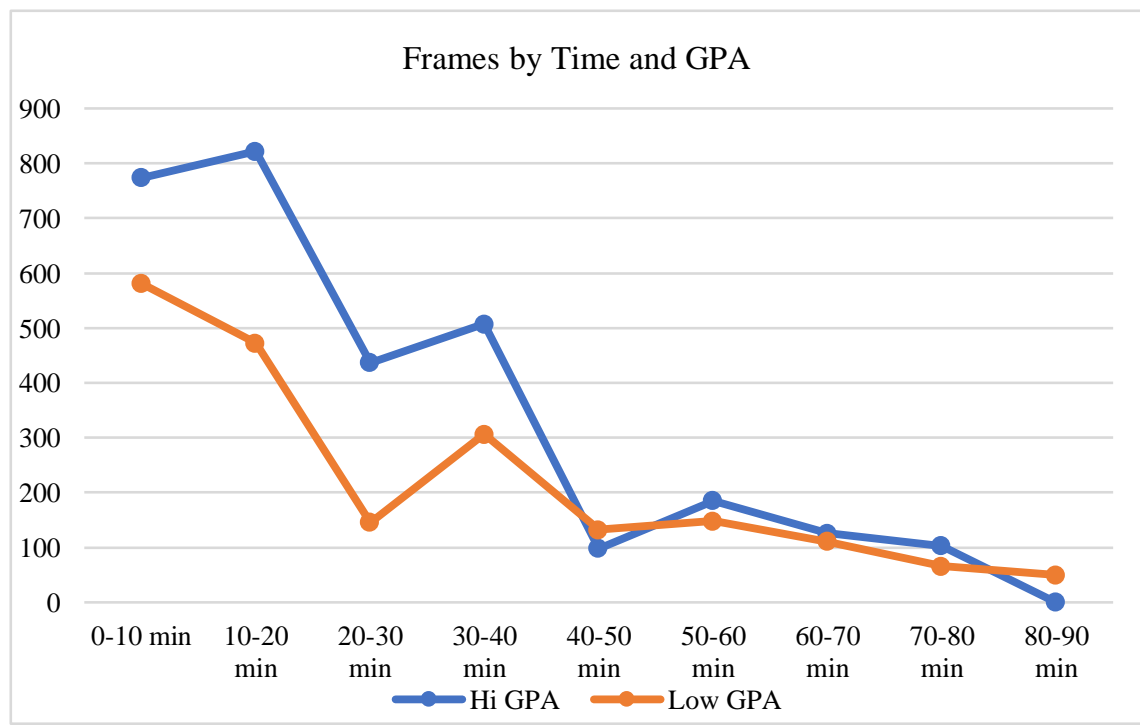

Figura 6. Las estudiantes con rendimientos altos y bajos y su atención visual.

\section{Estudiantes Extrovertidas e Introvertidas y su Atención Visual}

A diferencia del análisis expuesto en la sección 4.1, donde cada frame podía tener múltiples variables, por ejemplo, un maestro podía estar gesticulando y 
señalando al mismo tiempo, los análisis de las secciones 4.2 y 4.3 son diferentes en su naturaleza, debido a la relación entre las variables. Es decir, se pueden percibir las diferencias de atención visual de una estudiante que tiene un GPA alto o bajo y, además, se pueden percibir las diferencias de atención visual entre una estudiante introvertida y una de extrovertida, pero la relación entre estas variables es mutuamente excluyente. Con esto en mente, en los primeros 10 minutos de la interacción en el aula, 914 del total de 1355 frames surgieron de estudiantes introvertidas que se relacionaban visualmente con el profesor. Además, 441 frames de 1355 provienen de estudiantes extrovertidas. Esto significa que, en los primeros 10 minutos de la lección, las introvertidas tenían más del doble de compromiso y atención visuales con su profesor que las extrovertidas.

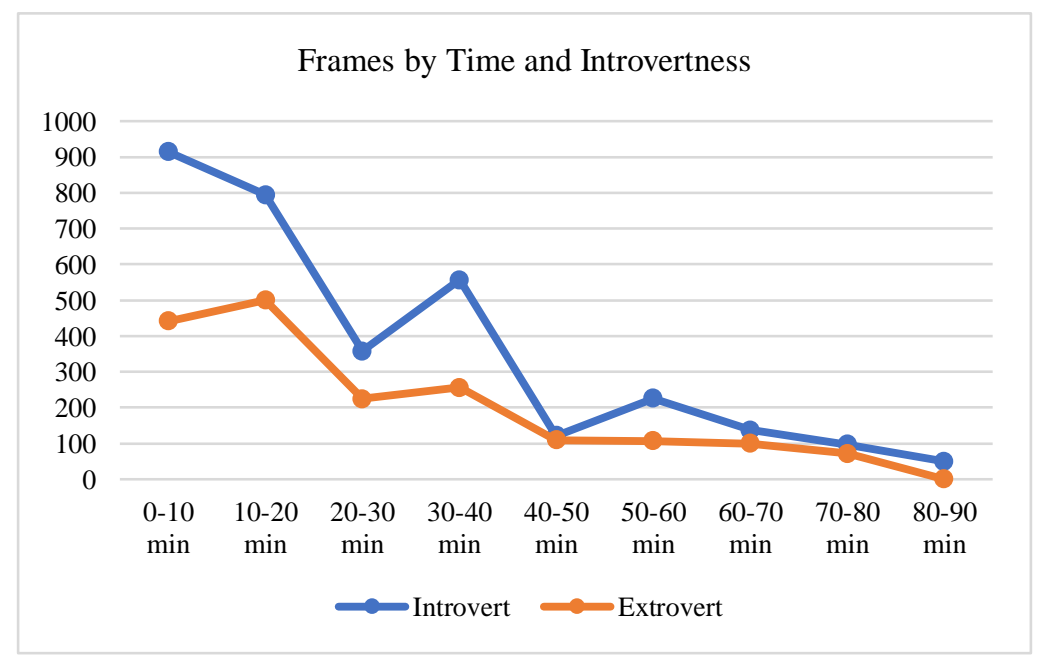

Figura 7. Estudiantes introvertidas y extrovertidas y su atención visual

\section{Observaciones Finales}

Este artículo reporta un estudio que se llevó a cabo en una escuela de educación primaria en Santiago de Chile, donde una muestra de 33 estudiantes de sexo femenino, seleccionadas al azar, llevaba una mini cámara de vídeo montado en sus gafas durante sus clases de matemáticas. Usamos el Google Images para buscar automática y objetivamente frames de las 
grabaciones en las que el profesor apareció en el campo visual de las estudiantes.

Los resultados de este estudio muestran que las estudiantes prestaron significativamente más atención visual en sus lecciones de matemáticas cuando el discurso del maestro estuvo acompañado de gestos, particularmente en los primeros 10 minutos de la lección. Las indicaciones del maestro parecían ser utilizadas como un instrumento para seguir motivando a las estudiantes. También, encontramos diferencias entre las estudiantes de alto y bajo rendimiento y cómo se involucraron visualmente durante el proceso de instrucción de su maestro en diferentes momentos de la lección. Además, se han encontrado diferencias entre cómo las alumnas introvertidas y las extrovertidas se involucraban visualmente mientras el maestro hacía gestos. En particular, se concluyó que las estudiantes introvertidas dedicaban más del doble de la atención a la instrucción de su maestro durante los primeros 10 minutos de la lección. En general, las alumnas introvertidas mantuvieron una atención visual ligeramente superior a lo largo de la lección que las extrovertidas.

Si bien los resultados de este estudio exploratorio muestran la importancia de la comunicación no-verbal (en particular, el uso de gestos realizados por los maestros) en los procesos interactivos entre profesor y alumno en el aula con el objetivo de implicar una mejora en los procesos de enseñanza y aprendizaje de las matemáticas, se concluye que los hallazgos de esta investigación están relacionados a un contexto muy particular, una muestra de 33 alumnas de dos escuelas de una región vulnerable en la ciudad de Santiago de Chile.

En ese sentido, se considera la importancia de que se realicen más investigaciones para analizar, de forma más amplia, la manera en que los estudiantes participan visualmente durante un proceso de instrucción matemática realizado por sus maestros (sean realizadas en contextos distintos o bien con una muestra más grande de sujetos participantes).

Además, independientemente de la experiencia del profesor, siempre es interesante cuestionar las formas, los estilos y la calidad de los mensajes que se transmiten de forma verbal y no verbal en la práctica profesional docente. Creemos que las optimizaciones de estos mensajes no verbales, muy sutiles y silenciosos pueden tener un efecto positivo directo, no solo con estudiantes con compromiso visual, sino también en el proceso de enseñanza y aprendizaje en general. Una recomendación y aplicación práctica es 
incorporar capacitación no verbal en los cursos de formación docente, tanto para maestros en formación como en servicio, a fin de crear conciencia sobre la función comunicativa del lenguaje no verbal, o bien, su incorporación en los desarrollos teóricos de la investigación en Educación Matemática, dado que los gestos y el lenguaje no verbal, son parte de los procesos interactivos que ocurren en una clase de matemáticas.

\section{Agradecimientos}

Los autores quieren agradecer el apoyo del proyecto "Uso del lesson study y la noción de idoneidad didáctica para el desarrollo de la competencia de análisis e intervención didáctica en la formación de profesores de matemáticas" (PGC2018-098603-B-I00) MINECO.

\section{Referencias}

Alibali, M. W. (1999). How children change their minds: Strategy change can be gradual or abrupt. Developmental Psychology, 35, 127-145. Alibali, M. W., \& Nathan, M. J. (2007). Teachers' gestures as a means of scaffolding students' understanding: Evidence from an early algebra lesson. In R. Goldman, R. Pea, B. Barron, \& S. J. Derry (Eds.), Video research in the learning sciences (pp. 349-365). Erlbaum.

Alibali, M. W., \& Nathan, M. J. (2012) Embodiment in mathematics teaching and learning: Evidence from learners' and teachers' gestures. Journal of the Learning Sciences, 21(2), 247-286. https://doi.org/10.1080/10508406.2011.611446

Andersen, J. F. (1978) The relationship between teacher immediacy and teaching effectiveness. [Doctoral Dissertation, West Virginia University].

Barakat, R. A. (1973). Arabic gestures. The Journal of Popular Culture, 6(4), 749-793. https://doi.org/10.1111/j.0022-3840.1973.00749.x

Bosch, M., \& Gascón, J. (2009). Aportaciones de la Teoría Antropológica de lo Didáctico a la formación del profesorado de matemáticas de secundaria. In M. T. G. María José González, Jesús Murillo. (Ed.), Investigación en Educación Matemática XIII (pp. 89-113). SEIEM.

Breda, A., Font, V., \& Pino-Fan, L. (2018). Criterios valorativos y normativos en la didáctica de las matemáticas: el caso del constructo 
idoneidad didáctica. Bolema, 32(60), 255-278.

https://doi.org/10.1590/1980-4415v32n60a13

Castellón, V. C. (2007). Students' speech and gesture: A communicative and strategic tool to convey and discuss mathematical concepts in a bilingual classroom. [Unpublished Doctoral Dissertation, University of California].

Catalán, J. (2018). Interpretación y cambio de Teorías Subjetivas. Editorial Universidad de La Serena.

Chevallard, Y. (1999). El análisis de las prácticas docentes en la teoría antropológica de los didáctico. Recherches en Didáctique des Mathématiques, 19(2), 221-266.

Collier, J. (1995). Photography and visual anthropology. In P. Hockings (ed.), Principles of Visual Anthropology, second edition (pp. 235 254). Mouton.

Corbell, A. (1997). Thumbs up in ancient Rome: "Pollex" as index. Memoirs of the American Academy in Rome, 42, 1-21. https://doi.org/10.2307/4238745

Ekman, P. (1976) Movements with precise meanings. Journal of Communication, 26(3), 14-26. https://doi.org/10.1111/j.14602466.1976.tb01898.x

Ekman, P., \& Friesen, W. V. (1969) Tools for the Analysis of Motion Picture Film or Video Tape. American Psychologist, 24(3), 240-243. https://doi.org/10.1037/h0028327

Farsani, D. (2015a). Deictic gestures as amplifiers in conveying aspects of mathematics register. In Proceedings of the $9^{\text {th }}$ Conference of European Society for Research in Mathematics Education, pp. 13821384. Prague, Czech Republic.

Farsani, D. (2015b). Making Multi-Modal Mathematical Meaning in Multilingual Classrooms. [Unpublished Doctoral dissertation, University of Birmingham].

Farsani, D. (2016). Complementary functions of learning mathematics in complementary schools. In P. C. Clarkson \& A. Halai (Eds.), Teaching \& Learning Mathematics in Multilingual Classrooms: Issues for policy, practice and teacher education (pp. 227-247). Sense Publishers.

Farsani, D., \& Mendes., J. (in press) Proxêmica e comunicação não verbal na interação em sala de aula. Psicologia Escolar e Educacional. 
240 Farsani, Breda \& Sala-Gestos del maestro y atención de las estudiantes

Galanti, G. A. (2004). Caring for Patients from Different Cultures. Third edition. University of Pennsylvania Press.

García, E. \& Hannula, H. (2015). Using gaze tracking technology to study student visual attention during teacher's presentation on board. In: Proceedings of the 9th Conference of European Society for Research in Mathematics Education. Prague, Czech Republic.

Godino, J. D., Batanero, C., \& Font, V. (2007). The onto-semiotic approach to research in mathematics education. ZDM- The International Journal of Mathematics Education, 39(1), 127-135. https://doi.org/10.1007/s11858-006-0004-1

Godino, J. D., Batanero, C., \& Font, V. (2019). The Onto-semiotic Approach: implications for the isteprescriptive character of didactics. For the Learning of Mathematics, 39(1), 37-42.

Gullberg, M. (1998). Gesture as a Communication Strategy in Second Language Discourse: A study of learners of French and Swedish. Lund University Press.

Hockings, P. (ed.) (1995). Principles of visual anthropology [2nd edition]. Mouton.

Kendon, A. (1983) Gesture and speech: How they interact. In J. M.

Wiemann \& R. P. Harrison (Eds.), Nonverbal interaction (pp. 13-45). Sage Publications.

Kendon, A. (1985). 'Behavioural Foundations for the Process of Frame Attunement in Face-to-Face Interaction'. In G. P. Ginsburg, M. Brenner, M. von Cranach (Eds.), Discovery Strategies in the Psychology of Action (pp. 229 253). Academic Press.

Kendon, A. (1997). Gesture. Annual Review of Anthropology, 26, 109-128. https://doi.org/10.1146/annurev.anthro.26.1.109

Knapp, M. L., \& Hall, J. A. (2006). Nonverbal communication in human interaction. Thompson Publishers.

Koechlin, B. (1992). Prolegomenon to the elaboration for a new discipline: Ethnogestics. In F. Poyatos (ed.), Advances in Nonverbal Communication: Social, Cultural, Esthetic and literary perspectives (pp. 59-76). John Benjamins.

Lemke, J. L. (1998) Multiplying meaning: visual and verbal semiotics in scientific text. In J. R. Martin, \& R. Veel (Eds.), Reading science (pp. 87-113). Routledge 
Maschietto, M., \& Bussi, M. G. B. (2005) Meaning construction through semiotic means: the case of the visual pyramid. In H. L. Chick, \& J. L. Vincent (Eds.), Proceedings of the 29th Conference of the International Group for the Psychology of Mathematics Education (pp. 313-320). Melbourne, Australia.

McNeill, D. (1992). Hand and mind: What gestures reveal about thought. The University of Chicago Press.

Núñez, R., \& Sweetser, E. (2006). With the future behind them: Convergent evidence from Aymara language and gesture in the crosslinguistic comparison of spatial construal of time. Cognitive Science, 30(3), 401-450. https://doi.org/10.1207/s15516709cog0000_62

Pier, E. L., \& Nathan, M. J. (2016). A review of mathematics and the body: Material engagements in the classroom. Journal for Research in Mathematics Education, 47(4), 223-227.

https://doi.org/10.5951/jresematheduc.47.4.0423

Pogue, L., \& Ahyun, K. (2006) The effect of teacher nonverbal immediacy and credibility on student motivation and affective learning. Communication Education, 55(3), 331-344. https://doi.org/10.1080/03634520600748623

Prieto, L. P., Sharma, K., \& Dillenbourg, P. (2015) Studying teacher orchestration load in technology-enhanced classrooms. Conole G., Klobučar T., Rensing C., Konert J., Lavoué E. (eds), Design for Teaching and Learning in a Networked World (pp. 268 -281). Springer International Publishing.

Raca, M., \& Dillenbourg, P. (2013) System for assessing classroom attention. In Proceedings of the Third International Conference on Learning Analytics and Knowledge (pp. 265 -269). https://doi.org/10.1145/2460296.2460351

Rogers, B. (2015). Classroom behaviour: A practical guide to effective teaching, behaviour management and colleague support. Sage Publication.

Roth, W. M. (2002) Gestures: Their role in teaching and learning. Review of Educational Research, 71(3), 365 - 392. https://doi.org/10.3102/00346543071003365

Sfard, A. (2009) What's all the fuss about gestures? A commentary. Educational Studies in Mathematics, 70(2), 191-200. https://doi.org/10.1007/s10649-008-9161-1 
242 Farsani, Breda \& Sala-Gestos del maestro y atención de las estudiantes

Sparhawk, C. M. P (1981). Contrastive-identificational features of Persian gesture. In A. Kendon (ed.), Nonverbal Communication, Interaction, and Gesture (pp.421-458). Mouton.

Sparhawk, C. M. P. (1976) Linguistic and gesture: An application of linguistic theory to the study of Persian emblems. Unpublished $\mathrm{PhD}$ Dissertation. Department of Linguistics, University of Michigan.

Streeck, J. (1988) The significance of gesture: How it is established. Papers in Pragmatics, 2(1/2), 60-83. https://doi.org/10.1075/iprapip.2.1$2.03 \mathrm{str}$

Danyal Farsani es investigador y profesor de Educación Matemática en la Universidad de Chile.

Adriana Breda es investigadora y profesora de Didáctica de las Matemáticas en la Universitat de Barcelona.

Gemma Sala Sebastià es investigadora y profesora de Didáctica de las Matemáticas en la Universitat Autònoma de Barcelona.

Dirección de contacto: La correspondencia directa sobre este artículo debe enviarse al autor. Dirección Postal: Centro de Investigación Avanzada en Educación, Universidad de Chile, Periodista José Carrasco Tapia 75, Santiago, Chile. Email: danyal.farsani@ciae.uchile.cl 